\title{
GENERALIZED ISOPERIMETRIC FVPS VIA CAPUTO APPROACH
}

\author{
By Amele TAÏEB AND Zoubir DAhmani
}

\begin{abstract}
In this paper, we study several fractional variational problems with functionals that contain $n$ unknown functions with their higher order Caputo derivatives and Riemann-Liouville integrals. We prove generalized fractional Euler-Lagrange equations. We also study an isoperimetric problem with multiple constraints, and we find the optimality conditions. Some examples are provided to illustrate the applications of the results.
\end{abstract}

1. Introduction and Preliminaries. It is known that the fractional calculus arises in various fields of applied sciences. For more details see, $\mathbf{1 8}$ 20, 25, 26]. Moreover, fractional differential equations play a central role in engineering sciences and applied mathematics in building mathematical models of many physical phenomena. See, for instance $[\mathbf{1}, \mathbf{1 0}, \mathbf{1 5}, \mathbf{1 6}, \mathbf{2 9}, \mathbf{3 0}, \mathbf{3 2}, \mathbf{3 3}$.

The calculus of variations is concerned with finding a function for which a given functional attains an extremum value. Books $\mathbf{9}, \mathbf{1 3}, \mathbf{1 7}, \mathbf{2 1}, \mathbf{2 2}, \mathbf{2 4}$ may serve as an introduction to the calculus of variations for mathematicians and scientists, and the reader can found interesting results in geometry and differential equations therein. The calculus of variations plays an important role in problems arising in classical mechanics, economics, electrical engineering, urban planning and other fields. For details, see $[\mathbf{1 4}, \mathbf{2 7}, \mathbf{3 4}, \mathbf{3 5}$ and the references therein. Some researchers study different fractional variational problems and several important research results have been obtained in $2-8,11,12,23,31,36$.

2000 Mathematics Subject Classification. 49K10, 26A33, 26B20.

Key words and phrases. Caputo derivative, Fractional calculus of variations, isoperimetric problems. 
The present paper is devoted to building necessary conditions of EulerLagrange type for fractional variational problem of the form:

$$
J\left(y_{1}, \ldots, y_{n}\right)=\int_{x_{0}}^{x_{1}}\left(L \circ \underset{\alpha_{k}^{j}}{\gamma_{k}}\left[y_{1}, \ldots, y_{n}\right]_{\beta_{k}^{j}}\right)(x) d x \rightarrow \text { extremum }
$$

where $\gamma_{k}>0, j-1<\alpha_{k}^{j}, \beta_{k}^{j}<j ; j, k=1,2, \ldots, n, n \in \mathbb{N}^{*}$ with $\mathbb{N}^{*}=\mathbb{N}-\{0\}$, $\left(y_{k}\right)_{k=1,2, \ldots, n} \in S:=\left\{\left(y_{1}, \ldots, y_{n}\right) \in C^{n}\left(\left[x_{0}, x_{1}\right]\right) ; y_{k}^{(i)}\left(x_{0}\right)=a_{k}^{i}, y_{k}^{(i)}\left(x_{1}\right)=\right.$ $\left.b_{k}^{i}, i=0,1, \ldots, n-1, n \in \mathbb{N}^{*}\right\}$, and

$$
\begin{aligned}
{ }_{\alpha_{k}^{j}}^{\gamma_{k}}\left[y_{1}, \ldots, y_{n}\right]_{\beta_{k}^{j}}:= & \left(x, y_{1}(x), \ldots, y_{n}(x),\right. \\
& { }_{x_{0}}^{C} D_{x}^{\alpha_{1}^{1}} y_{1}(x),{ }_{x_{0}}^{C} D_{x}^{\alpha_{1}^{2}} y_{1}(x) \ldots,{ }_{x_{0}}^{C} D_{x}^{\alpha_{1}^{n}} y_{1}(x), \\
& \ldots \\
& { }_{x_{0}}^{C} D_{x}^{\alpha_{n}^{1}} y_{n}(x),{ }_{x_{0}}^{C_{0}} D_{x}^{\alpha_{n}^{2}} y_{n}(x) \ldots,{ }_{x_{0}}^{C_{0}} D_{x}^{\alpha_{n}^{n}} y_{n}(x), \\
& { }_{x}^{C} D_{x_{1}}^{\beta_{1}^{1}} y_{1}(x),{ }_{x}^{C} D_{x_{1}}^{\beta_{1}^{2}} y_{1}(x), \ldots,{ }_{x}^{C} D_{x_{1}}^{\beta_{1}^{n}} y_{1}(x), \\
& \ldots \\
& { }_{x}^{C} D_{x_{1}}^{\beta_{n}^{1}} y_{n}(x),{ }_{x}^{C} D_{x_{1}}^{\beta_{n}^{2}} y_{n}(x), \ldots,{ }_{x}^{C} D_{x_{1}}^{\beta_{n}^{n}} y_{n}(x), \\
& \left.x_{0} I_{x}^{\gamma_{1}} y_{1}(x), \ldots,{ }_{x_{0}} I_{x}^{\gamma_{n}} y_{n}(x)\right) .
\end{aligned}
$$

We will consider the fractional isoperimetric problem that consists in maximizing (or minimizing) the above functional subject to the given boundary conditions and $m$ fractional integral constraints:

$$
I_{q}\left(y_{1}, \ldots, y_{n}\right)=\int_{x_{0}}^{x_{1}}\left(F_{q} \circ \underset{\alpha_{k}^{j}}{\gamma_{k}}\left[y_{1}, \ldots, y_{n}\right]_{\beta_{k}^{j}}\right)(x) d x=l_{q},
$$

where $q=1,2, \ldots m, m<n$, and $l_{q} \in \mathbb{R}$.

Finally, we will present the optimality conditions for a functions-time pair $\left(\left(y_{k}\right)_{k=1, \ldots, n}, T\right) \in\left\{\left(C^{n}\left(\left[x_{0}, x_{1}\right]\right),\left[x_{0}, x_{1}\right]\right): y_{k}^{(i)}\left(x_{0}\right)=a_{k}^{i}\right\}$ to be an optimal solution to:

$$
J^{*}\left(y_{1}, \ldots, y_{n}, T\right)=\int_{x_{0}}^{T}\left(L^{*} \circ \alpha_{k}^{j}\left[y_{1}, \ldots, y_{n}\right]\right) d x
$$


where

$$
\begin{aligned}
\alpha_{k}^{j}\left[y_{1}, \ldots, y_{n}\right]:= & \left(x, y_{1}(x), \ldots, y_{n}(x),\right. \\
& { }_{x_{0}}^{C} D_{x}^{\alpha_{1}^{1}} y_{1}(x),{ }_{x_{0}}^{C} D_{x}^{\alpha_{1}^{2}} y_{1}(x), \ldots,{ }_{x_{0}}^{C} D_{x}^{\alpha_{1}^{n}} y_{1}(x), \\
& \cdots \\
& \left.{ }_{x_{0}}^{C} D_{x}^{\alpha_{n}^{1}} y_{n}(x),{ }_{x_{0}}^{C_{0}} D_{x}^{\alpha_{n}^{2}} y_{n}(x), \ldots,{ }_{x_{0}} D_{x}^{\alpha_{n}^{n}} y_{n}(x)\right) .
\end{aligned}
$$

We consider the both free and fixed end-point problems.

To the best of our knowledge, there are no papers dealing with this kind of high dimensional variational problems.

Now, we present some basic definitions and lemmas that we need to prove our main results.

Definition 1. $[\mathbf{1 9}, \mathbf{2 5}, 26$ The left and right Riemann-Liouville fractional integral operators of order $\alpha>0$ for an integrable function $y$ on $\left[x_{0}, x_{1}\right]$ are defined by:

$$
x_{0} I_{x}^{\alpha} y(x)=\frac{1}{\Gamma(\alpha)} \int_{x_{0}}^{x}(x-t)^{\alpha-1} y(t) d t
$$

and

$$
{ }_{x} I_{x_{1}}^{\alpha} y(x)=\frac{1}{\Gamma(\alpha)} \int_{x}^{x_{1}}(t-x)^{\alpha-1} y(t) d t,
$$

respectively, where $\Gamma(\alpha):=\int_{0}^{\infty} e^{-u} u^{\alpha-1} d u$.

Definition 2. $\mathbf{1 9}, \mathbf{2 5}, \mathbf{2 6}$ The left and right Riemann-Liouville fractional derivative operators of order $\alpha>0$ for a function $y:\left[x_{0}, x_{1}\right] \rightarrow \mathbb{R}$ are defined as:

$$
{ }_{x_{0}}^{R L} D_{x}^{\alpha} y(x)=\frac{1}{\Gamma(n-\alpha)}\left(\frac{d}{d x}\right)^{(n)} \int_{x_{0}}^{x}(x-t)^{n-\alpha-1} y(t) d t
$$

and

$$
{ }_{x}^{R L} D_{x_{1}}^{\alpha} y(x)=\frac{1}{\Gamma(n-\alpha)}\left(-\frac{d}{d x}\right)^{(n)} \int_{x}^{x_{1}}(t-x)^{n-\alpha-1} y(t) d t,
$$

respectively, for $n-1<\alpha<n, n \in \mathbb{N}^{*}$.

Definition 3. 19, 25, 26 The left and right Caputo fractional derivative operators of order $\alpha>0$ for a function $y:\left[x_{0}, x_{1}\right] \rightarrow \mathbb{R}$, which is at least $n$-times differentiable are defined as:

$$
{ }_{x_{0}}^{C} D_{x}^{\alpha} y(x)=\frac{1}{\Gamma(n-\alpha)} \int_{x_{0}}^{x}(x-t)^{n-\alpha-1} y^{(n)}(t) d t,
$$


and

$$
{ }_{x}^{C} D_{x_{1}}^{\alpha} y(x)=\frac{1}{\Gamma(n-\alpha)} \int_{x}^{x_{1}}(t-x)^{n-\alpha-1}(-1)^{n} y^{(n)}(t) d t,
$$

respectively, for $n-1<\alpha<n, n \in \mathbb{N}^{*}$.

Lemma 4. [19, 25, 26, 28 Assume that $n-1<\alpha<n, n \in \mathbb{N}^{*}$ and $f$ is of class $C^{n}$ on $\left[x_{0}, x_{1}\right]$. Then its left and right Caputo derivatives of order $\alpha$ are continuous on $\left[x_{0}, x_{1}\right]$.

LEMma 5. $\quad$ 13, 24 Let $f:\left[x_{0}, x_{1}\right] \rightarrow \mathbb{R}$ be a continuous function such that:

$$
\int_{x_{0}}^{x_{1}} f(x) \sigma(x) d x=0
$$

holds for every $\sigma \in C^{n}\left(\left[x_{0}, x_{1}\right]\right), n \geq 0$, satisfying $\sigma\left(x_{0}\right)=\sigma\left(x_{1}\right)=0$. Then, $f(x)=0$ on $\left[x_{0}, x_{1}\right]$.

Lemma 6. 28 Let $\alpha>0, p, q \geq 1$, and $\frac{1}{p}+\frac{1}{q} \leq 1+\alpha$. If $g \in L^{p}\left(\left[x_{0}, x_{1}\right]\right)$ and $f \in L^{q}\left(\left[x_{0}, x_{1}\right]\right)$, then

$$
\int_{x_{0}}^{x_{1}} g(x){ }_{x_{0}} I_{x}^{\alpha} f(x) d x=\int_{x_{0}}^{x_{1}} f(x){ }_{x} I_{x_{1}}^{\alpha} g(x) d x .
$$

LEMma 7. 28 Let $n-1<\alpha<n, f, g:\left[x_{0}, x_{1}\right] \rightarrow \mathbb{R}, f$ be of class $C^{n}$, $g$ and ${ }_{x} D_{x_{1}}^{\alpha} g$ be continuous functions on $\left[x_{0}, x_{1}\right]$. Then,

$$
\begin{aligned}
& \int_{x_{0}}^{x_{1}} g(x){ }_{x_{0}}^{C} D_{x}^{\alpha} f(x) d x=\int_{x_{0}}^{x_{1}} f(x){ }_{x}^{R L} D_{x_{1}}^{\alpha} g(x) d x \\
& +\sum_{j=0}^{n-1}\left[{ }_{x}^{R L} D_{x_{1}}^{\alpha+j-n} g(x){ }_{x}^{R L} D_{x_{1}}^{n-j-1} f(x)\right]_{x_{0}}^{x_{1}}, \\
& \int_{x_{0}}^{x_{1}} g(x){ }_{x}^{C} D_{x_{1}}^{\alpha} f(x) d x=\int_{x_{0}}^{x_{1}} f(x){ }_{x_{0}}^{R L} D_{x}^{\alpha} g(x) d x \\
& +\sum_{j=0}^{n-1}\left[(-1)^{(n+j)}{ }_{x_{0}}^{R L} D_{x}^{\alpha+j-n} g(x){ }_{x_{0}}^{R L} D_{x}^{n-j-1} f(x)\right]_{x_{0}}^{x_{1}} .
\end{aligned}
$$

REMARK 8. If $f\left(x_{0}\right)=f\left(x_{1}\right)=0$, then

$$
\int_{x_{0}}^{x_{1}} g(x){ }_{x_{0}}^{C_{0}} D_{x}^{\alpha} f(x) d x=\int_{x_{0}}^{x_{1}} f(x){ }_{x}^{R L} D_{x_{1}}^{\alpha} f(x) d x
$$

and

$$
\int_{x_{0}}^{x_{1}} g(x){ }_{x}^{C} D_{x_{1}}^{\alpha} f(x) d x=\int_{x_{0}}^{x_{1}} f(x){ }_{x_{0}}^{R L} D_{x}^{\alpha} f(x) d x .
$$


2. Necessary Optimality Conditions. In this section, we present necessary optimality conditions of Euler-Lagrange type for variational problems with functional involving $n$ unknown functions with their higher order left and right Caputo fractional derivatives and fractional integrals.

Define the nonlinear operator ${ }_{\alpha_{k}^{j}}^{\gamma_{k}}\left[y_{1}, \ldots, y_{n}\right]_{\beta_{k}^{j}}$ by:

$$
\begin{aligned}
{ }_{\alpha_{k}^{j}}^{\gamma_{k}}\left[y_{1}, \ldots, y_{n}\right]_{\beta_{k}^{j}}:= & \left(x, y_{1}(x), \ldots, y_{n}(x),\right. \\
& { }_{x_{0}}^{C} D_{x}^{\alpha_{1}^{1}} y_{1}(x), \ldots,{ }_{x_{0}}^{C} D_{x}^{\alpha_{1}^{n}} y_{1}(x), \\
& \ldots \\
& { }_{x_{0}} D_{x}^{\alpha_{n}^{1}} y_{n}(x), \ldots,{ }_{x_{0}}^{C} D_{x}^{\alpha_{n}^{n}} y_{n}(x), \\
& { }_{x}^{C} D_{x_{1}}^{\beta_{1}^{1}} y_{1}(x), \ldots,{ }_{x}^{C} D_{x_{1}^{1}}^{\beta_{1}^{n}} y_{1}(x), \\
& \ldots \\
& { }_{x}^{C} D_{x_{1}}^{\beta_{n}^{1}} y_{n}(x), \ldots,{ }_{x}^{C} D_{x_{1}}^{\beta_{n}^{n}} y_{n}(x), \\
& \left.{ }_{x_{0}} I_{x}^{\gamma_{1}} y_{1}(x), \ldots,{ }_{x_{0}}{ }_{x}^{\gamma_{n}} y_{n}(x)\right),
\end{aligned}
$$

where $\gamma_{k}>0, j-1<\alpha_{k}^{j}, \beta_{k}^{j}<j, j, k=1,2, \ldots, n, n \in \mathbb{N}^{*}$.

Define the set $S$ by:

$$
\begin{aligned}
S:= & \left\{\left(y_{1}, \ldots, y_{n}\right) \in C^{n}\left(\left[x_{0}, x_{1}\right]\right) ; y_{k}^{(i)}\left(x_{0}\right)=a_{k}^{i}, y_{k}^{(i)}\left(x_{1}\right)=b_{k}^{i}\right\}, \\
& k=1,2, \ldots, n, i=0,1, \ldots, n-1, n \in \mathbb{N}^{*} .
\end{aligned}
$$

Let $J$ be a functional of the form:

$$
J\left(y_{1}, \ldots, y_{n}\right)=\int_{x_{0}}^{x_{1}}\left(L \circ \underset{\alpha_{k}^{j}}{\gamma_{k}}\left[y_{1}, \ldots, y_{n}\right]_{\beta_{k}^{j}}\right)(x) d x,
$$

and consider the problem of finding functions $\left(y_{k}\right)_{k=1,2, \ldots, n} \in S$ that maximize or minimize the functional $J$.

For the sake of convenience, we denote by $\partial_{p} L, p=1, \ldots, 2 n^{2}+2 n+1$ the partial derivative of the function $L:\left[x_{0}, x_{1}\right] \times \mathbb{R}^{2 n^{2}+2 n+1} \rightarrow \mathbb{R}$ with respect to its $p$-th argument. Also, we assume that:

$\left(H_{1}\right)$ : The function $L$ is of class $C^{1}$ with respect to all of its arguments.

$\left(H_{2}\right):\left(\partial_{p} L\right)_{p=n+2, \ldots, n^{2}+n+1}$ has continuous right Riemann-Liouville fractional derivative of order $\alpha_{k}^{j}, j, k=1,2, \ldots, n$, respectively.

$\left(H_{3}\right):\left(\partial_{p} L\right)_{p=n^{2}+n+2, \ldots, 2 n^{2}+n+1}$ has continuous left Riemann-Liouville fractional derivative of order $\beta_{k}^{j}, j, k=1,2, \ldots, n$, respectively.

Theorem 9. Assume that the functional of the form given by Eq. 15) has an extremum in $S$ at $\left(y_{k}\right)_{k=1,2, \ldots, n}$. Then for all $x \in\left[x_{0}, x_{1}\right]$, the functions 
$\left(y_{k}\right)_{k=1,2, \ldots, n}$ satisfy the generalized fractional Euler-Lagrange equations:

$$
\begin{aligned}
\partial_{p} L & +\sum_{j=1}^{n}{ }_{x}^{R L} D_{x}^{\alpha_{p-1}^{j}} \partial_{n(p-1)+j+1} L \\
& +\sum_{j=1}^{n}{ }_{x_{0}}^{R L} D_{x}^{\beta_{p-1}^{j}} \partial_{n(p-1)+n^{2}+j+1} L+{ }_{x} I_{x_{1}}^{\gamma_{p-1}} \partial_{2 n^{2}+n+p} L \\
= & 0
\end{aligned}
$$

where $p=2, \ldots, n+1$.

Proof. Define $S^{*}$ by:

$$
S^{*}:=\left\{\sigma_{k} \in C^{n}\left(\left[x_{0}, x_{1}\right]\right): \sigma_{k}^{(i)}\left(x_{0}\right)=\sigma_{k}^{(i)}\left(x_{1}\right)=0, i=0,1, \ldots, n-1\right\} .
$$

For $\epsilon_{k}>0, \sigma_{k} \in S^{*}$, let $y_{k}+\epsilon \sigma_{k}$ be the variation of $y_{k}$, with $\left(y_{k}\right)_{k=1,2, \ldots n} \in S$ is solution for the functional in Eq. (15).

Then,

$$
J\left(\epsilon_{1}, \ldots, \epsilon_{n}\right)=\int_{x_{0}}^{x_{1}}\left(L \circ \underset{\alpha_{k}^{j}}{\gamma_{k}}\left[y_{1}+\epsilon_{1} \sigma_{1}, \ldots, y_{n}+\epsilon_{n} \sigma_{n}\right]_{\beta_{k}^{j}}\right)(x) d x .
$$

Since $J\left(\epsilon_{1}, \ldots, \epsilon_{n}\right)$ attains an extreme value at $\left(\epsilon_{1}, \ldots, \epsilon_{n}\right)=(0, \ldots, 0)$, we get:

$$
\left(\frac{\partial J}{\partial \epsilon_{k}}\right)\left(\epsilon_{k}=0\right)=0, k=1,2, \ldots, n .
$$

We know that the linearity of ${ }_{x_{0}}^{C} D_{x}^{\alpha_{k}^{j}}$ and ${ }_{x}^{C} D_{x_{1}}^{\beta_{k}^{j}}$ implies

$$
{ }_{x_{0}}^{C} D_{x}^{\alpha_{k}^{j}}\left(y_{k}+\epsilon_{k} \sigma_{k}\right)(x)={ }_{x_{0}}^{C} D_{x}^{\alpha_{k}^{j}} y_{k}+\epsilon_{k}{ }_{x_{0}}^{C} D_{x}^{\alpha_{k}^{j}} \sigma_{k}(x),
$$

and

$$
{ }_{x}^{C} D_{x_{1}}^{\beta_{k}^{j}}\left(y_{k}+\epsilon_{k} \sigma_{k}\right)(x)={ }_{x}^{C} D_{x_{1}}^{\beta_{k}^{j}} y_{k}+\epsilon_{k}{ }_{x}^{C} D_{x_{1}}^{\beta_{k}^{j}} \sigma_{k}(x) .
$$

Consequently, Eq. 19) implies

$$
\begin{aligned}
\int_{x_{0}}^{x_{1}} & \left(\partial_{p} L \sigma_{p-1}(x)+\sum_{j=1}^{n} \partial_{n(p-1)+j+1} L{ }_{x_{0}}^{C} D_{x}^{\alpha_{p-1}^{j}} \sigma_{p-1}(x)\right. \\
(22) & \left.+\sum_{j=1}^{n} \partial_{n(p-1)+n^{2}+j+1} L{ }_{x}^{C} D_{x_{1}}^{\beta_{p-1}^{j}} \sigma_{p-1}(x)+\partial_{2 n^{2}+n+p} L{ }_{x_{0}}{ }_{x}^{\gamma_{p-1}} \sigma_{p-1}(x)\right) d x \\
& =0, \quad p=2, \ldots, n+1 .
\end{aligned}
$$


Integrating by parts as in Eqs. (8), (9) and (10), we get

$$
\begin{aligned}
& \int_{x_{0}}^{x_{1}}\left(\partial_{p} L \sigma_{p-1}(x)+\sigma_{p-1}(x) \sum_{j=1}^{n}{ }_{x}^{R L} D_{x_{1}}^{\alpha_{p-1}^{j}} \partial_{n(p-1)+j+1} L\right. \\
& \left.(23) \quad+\sigma_{p-1}(x) \sum_{j=1}^{n}{ }_{x_{0}}^{R L} D_{x}^{\beta_{p-1}^{j}} \partial_{n(p-1)+n^{2}+j+1} L+\sigma_{p-1}(x){ }_{x} I_{x_{1}}^{\gamma_{p-1}} \partial_{2 n^{2}+n+p} L\right) d x \\
& \quad=0, \quad p=2, \ldots, n+1 .
\end{aligned}
$$

Thus,

$$
\begin{aligned}
\int_{x_{0}}^{x_{1}} & \left(\partial_{p} L+\sum_{j=1}^{n}{ }_{x}^{R L} D_{x_{1}}^{\alpha_{p-1}^{j}} \partial_{n(p-1)+j+1} L\right. \\
& \left.+\sum_{j=1}^{n}{ }_{x_{0}}^{R L} D_{x}^{\beta_{p-1}^{j}} \partial_{n(p-1)+n^{2}+j+1} L+{ }_{x} I_{x_{1}}^{\gamma_{p-1}} \partial_{2 n^{2}+n+p} L\right) \sigma_{p-1}(x) d x \\
& =0, \quad p=2, \ldots, n+1 .
\end{aligned}
$$

It follows from Lemma 5 that

$$
\begin{aligned}
\partial_{p} L & +\sum_{j=1}^{n}{ }_{x}^{R L} D_{x_{1}}^{\alpha_{p-1}^{j}} \partial_{n(p-1)+j+1} L \\
& +\sum_{j=1}^{n}{ }_{x_{0}}^{R L} D_{x}^{\beta_{p-1}^{j}} \partial_{n(p-1)+n^{2}+j+1} L+{ }_{x} I_{x_{1}}^{\gamma_{p-1}} \partial_{2 n^{2}+n+p} L \\
& =0, \quad p=2, \ldots, n+1 .
\end{aligned}
$$

Equation 25 is the fractional Euler-Lagrange equations for the variational problem considered. This completes the proof.

Example 10. Consider the following problem:

$$
\begin{aligned}
J\left(y_{1}, y_{2}\right)= & \int_{-1}^{1}\left(2 x^{2}+y_{1}^{2}+2 y_{2}^{2}+\left({ }_{-1}^{C} D_{x}^{\frac{2}{3}} y_{1}\right)^{2}+\frac{1}{2}\left({ }_{-1}^{C} D_{x}^{\frac{4}{3}} y_{1}\right)^{2}\right. \\
& +3\left({ }_{-1}^{C} D_{x}^{\frac{3}{5}} y_{2}\right)^{2}+2\left({ }_{-1}^{C} D_{x}^{\frac{5}{3}} y_{2}\right)^{2}-\left({ }_{x}^{C} D_{1}^{\frac{1}{2}} y_{1}\right)-\left({ }_{x}^{C} D_{1}^{\frac{3}{2}} y_{1}\right) \\
& \left.+\left({ }_{x}^{C} D_{1}^{\frac{3}{5}} y_{2}\right)+\left({ }_{x}^{C} D_{1}^{\frac{8}{5}} y_{2}\right)+{ }_{-1} I_{x}^{\frac{7}{2}} y_{1}+2{ }_{-1} I_{x}^{\frac{10}{3}} y_{2}\right) d x,
\end{aligned}
$$

with

$$
\begin{aligned}
& y_{1}(-1)=1, \quad y_{1}^{\prime}(-1)=\sqrt{2}, \quad y_{1}(1)=2 \sqrt{2}, \quad y_{1}^{\prime}(1)=-1, \\
& y_{2}(-1)=0, \quad y_{2}^{\prime}(-1)=2, \quad y_{2}(1)=-1, \quad y_{2}^{\prime}(1)=\frac{1}{3} \text {. }
\end{aligned}
$$


Here, $n=2, x_{0}=-1, x_{1}=1, \alpha_{1}^{1}=\frac{2}{3}, \alpha_{1}^{2}=\frac{4}{3}, \alpha_{2}^{1}=\frac{3}{5}, \alpha_{2}^{2}=\frac{5}{3}, \beta_{1}^{1}=\frac{1}{2}$, $\beta_{1}^{2}=\frac{3}{2}, \beta_{2}^{1}=\frac{3}{5}, \beta_{2}^{2}=\frac{8}{5}, \gamma_{1}=\frac{7}{2}, \gamma_{2}=\frac{10}{3}$, and

$$
\begin{aligned}
L= & 2 x^{2}+y_{1}^{2}+2 y_{2}^{2} \\
& +\left({ }_{-1}^{C} D_{x}^{\frac{2}{3}} y_{1}\right)^{2}+\frac{1}{2}\left({ }_{-1}^{C} D_{x}^{\frac{4}{3}} y_{1}\right)^{2}+3\left({ }_{-1}^{C} D_{x}^{\frac{3}{5}} y_{2}\right)^{2} \\
& +2\left({ }_{-1}^{C} D_{x}^{\frac{5}{3}} y_{2}\right)^{2}-\left({ }_{x}^{C} D_{1}^{\frac{1}{2}} y_{1}\right)-\left({ }_{x}^{C} D_{1}^{\frac{3}{2}} y_{1}\right) \\
& +\left({ }_{x}^{C} D_{1}^{\frac{3}{5}} y_{2}\right)+\left({ }_{x}^{C} D_{1}^{\frac{8}{5}} y_{2}\right)+{ }_{-1} I_{x}^{\frac{7}{2}} y_{1}+2{ }_{-1} I_{x}^{\frac{10}{3}} y_{2} .
\end{aligned}
$$

The extremes for $J$ must satisfy the fractional Euler-Lagrange equations given in Eq. (16). So, we get

$$
\begin{gathered}
2 y_{1}+2{ }_{x}^{R L} D_{1}^{\frac{2}{3}}\left({ }_{-1}^{C} D_{x}^{\frac{2}{3}} y_{1}\right)+{ }_{x}^{R L} D_{1}^{\frac{4}{3}}\left({ }_{-1}^{C} D_{x}^{\frac{4}{3}} y_{1}\right) \\
-{ }_{-1}^{R L} D_{x}^{\frac{1}{2}}(1)-{ }_{-1}^{R L} D_{x}^{\frac{3}{2}}(1)+{ }_{x} I_{1}^{\frac{7}{2}}(1)=0,
\end{gathered}
$$

and

$$
\begin{gathered}
4 y_{2}+6{ }_{x}^{R L} D_{1}^{\frac{3}{5}}\left({ }_{-1}^{C} D_{x}^{\frac{3}{5}} y_{2}\right)+4{ }_{x}^{R L} D_{1}^{\frac{5}{3}}\left({ }_{-1}^{C} D_{x}^{\frac{5}{3}} y_{2}\right) \\
+{ }_{-1}^{R L} D_{x}^{\frac{3}{5}}(1)+{ }_{-1}^{R L} D_{x}^{\frac{8}{5}}(1)+{ }_{x} I_{1}^{\frac{10}{3}}(2)=0 .
\end{gathered}
$$

3. Generalized Isoperimetric Problems. We are now interested in finding the functions $\left(y_{k}\right)_{k=1,2, \ldots, n} \in S$ for which the functional given by Eq. (15), subject to the following fractional isoperimetric constraints:

$$
\begin{gathered}
I_{1}\left(y_{1}, \ldots, y_{n}\right)=\int_{x_{0}}^{x_{1}}\left(F_{1} \circ \underset{\alpha_{k}^{j}}{\gamma_{k}}\left[y_{1}, \ldots, y_{n}\right]_{\beta_{k}^{j}}\right)(x) d x=l_{1}, \\
I_{2}\left(y_{1}, \ldots, y_{n}\right)=\int_{x_{0}}^{x_{1}}\left(F_{2} \circ{ }_{\alpha_{k}^{j}}^{\gamma_{k}}\left[y_{1}, \ldots, y_{n}\right]_{\beta_{k}^{j}}\right)(x) d x=l_{2}, \\
\cdots \\
I_{m}\left(y_{1}, \ldots, y_{n}\right)=\int_{x_{0}}^{x_{1}}\left(F_{m} \circ{ }_{\alpha_{k}^{j}}^{\gamma_{k}}\left[y_{1}, \ldots, y_{n}\right]_{\beta_{k}^{j}}\right)(x) d x=l_{m}, \\
l_{q} \in \mathbb{R}, \quad q=1,2, \ldots, m, m<n .
\end{gathered}
$$

has an extremum.

Similarly, for all $q=1,2, \ldots m$, we denote by $\partial_{p} F_{q}, p=1, \ldots, 2 n^{2}+2 n+1$ the partial derivative of the function $F_{q}:\left[x_{0}, x_{1}\right] \times \mathbb{R}^{2 n^{2}+2 n+1} \rightarrow \mathbb{R}$ with respect to its $p$-th argument.

We also suppose that: 
$\left(H_{4}\right)$ : For each $q=1,2, \ldots m$, the function $F_{q}$ is of class $C^{1}$ with respect to all of its arguments.

$\left(H_{5}\right):\left(\partial_{p} F_{q}\right)_{p=n+2, \ldots, n^{2}+n+1}$ has continuous right Riemann-Liouville fractional derivatives of order $\alpha_{k}^{j}, j, k=1,2, \ldots, n$, respectively.

$\left(H_{6}\right):\left(\partial_{p} F_{q}\right)_{p=n^{2}+n+2, \ldots, 2 n^{2}+n+1}$ has continuous left Riemann-Liouville fractional derivatives of order $\beta_{k}^{j}, j, k=1,2, \ldots, n$, respectively.

ThEOREM 11. Suppose that $\left(y_{k}\right)_{k=1,2, \ldots, n} \in S$ is an extremizer of the functional given by Eq. (15), such that $I_{q}\left(y_{1}, \ldots, y_{n}\right)=l_{q}, q=1,2, \ldots, m$, $m<n$. If $\left(y_{k}\right)_{k=1,2, \ldots, n}$ is not an extremizer of $I_{q}$, then there exist constants $\left(\omega_{q}\right)_{q=1,2, \ldots, m}$ satisfying

$$
\begin{aligned}
\partial_{p} E & +\sum_{j=1}^{n}{ }_{x}^{R L} D_{x_{1}}^{\alpha_{p-1}^{j}} \partial_{n(p-1)+j+1} E \\
& +\sum_{j=1}^{n}{ }_{x_{0}}^{R L} D_{x}^{\beta_{p-1}^{j}} \partial_{n(p-1)+n^{2}+j+1} E+{ }_{x} I_{x_{1}}^{\gamma_{p-1}} \partial_{2 n^{2}+n+p} E=0,
\end{aligned}
$$

for all $x \in\left[x_{0}, x_{1}\right]$, where $p=2, \ldots, n+1$, and $E=L-\sum_{q=1}^{m} \omega_{q} F_{q}$.

Proof. Suppose that $\left(y_{k}\right)_{k=1,2, \ldots, n} \in S$ is a solution to the functional given in Eq. (15), such that $I_{q}\left(y_{1}, \ldots, y_{n}\right)=l_{q}, q=1,2, \ldots, m, m<n$, and $\left(y_{k}\right)_{k=1,2, \ldots, n}$ is not an extremizer of $I_{q}$.

Let $\epsilon_{k}^{t}>0, \sigma_{k}^{t} \in S^{*}, k=1,2, \ldots, n, t=1,2, \ldots, m+1, m<n$, and $y_{k}+\sum_{t=1}^{m+1} \epsilon_{k}^{t} \sigma_{k}^{t}$ be the variation of $y_{k}$. Then, we consider

$$
\begin{aligned}
J & \left(\epsilon_{1}^{1}, \ldots, \epsilon_{n}^{1}, \ldots, \epsilon_{1}^{m+1}, \ldots, \epsilon_{n}^{m+1}\right) \\
& =\int_{x_{0}}^{x_{1}}\left(L \circ{ }_{\alpha_{k}^{j}}^{\gamma_{k}}\left[y_{1}+\sum_{t=1}^{m+1} \epsilon_{1}^{t} \sigma_{1}^{t}, \ldots, y_{n}+\sum_{t=1}^{m+1} \epsilon_{n}^{t} \sigma_{n}^{t}\right]{ }_{\beta_{k}^{j}}\right)(x) d x,
\end{aligned}
$$

and

$$
\begin{aligned}
& I_{q}^{*}\left(\epsilon_{1}^{1}, \ldots, \epsilon_{n}^{1}, \ldots, \epsilon_{1}^{m+1}, \ldots, \epsilon_{n}^{m+1}\right) \\
& \quad=\int_{x_{0}}^{x_{1}}\left(F_{q} \circ{ }_{\alpha_{k}^{j}}^{\gamma_{k}}\left[y_{1}+\sum_{t=1}^{m+1} \epsilon_{1}^{t} \sigma_{1}^{t}, \ldots, y_{n}+\sum_{t=1}^{m+1} \epsilon_{n}^{t} \sigma_{n}^{t}\right]_{\beta_{k}^{j}}\right)(x) d x-l_{q} .
\end{aligned}
$$

The assumption that $\left(y_{k}\right)_{k=1,2, \ldots, n}$ is not an extremizer of $I_{q}$, means that there exists a family of functions $\left(\sigma_{k}^{t}\right)_{k=1,2, \ldots, n}^{t=2, \ldots, m+1}$, where

$$
\left.\left(\frac{\partial I_{q}^{*}}{\partial \epsilon_{p-1}^{t}}\right)\right|_{\left(\epsilon_{1}^{1}=\cdots=\epsilon_{1}^{m+1}=0, \ldots, \epsilon_{n}^{1}=\cdots=\epsilon_{n}^{m+1}=0\right)} \neq 0,
$$

for each $t=2, \ldots, m+1, k=1, \ldots, n, p=2, \ldots, n+1$. 
Also, by Eq. (34), we get $I_{q}^{*}(0, \ldots, 0)=0, q=1,2, \ldots, m$, for all $\epsilon_{k}^{t}=0$, $k=1,2, \ldots, n, t=1,2, \ldots, m+1$.

Since $I_{q}^{*}(0, \ldots, 0)=0, q=1,2, \ldots, m$, applying implicit function theorem, there exists a family of functions $\left(\epsilon_{k}^{t}\right)(\cdot), k=1,2, \ldots, n, t=2, \ldots, m+1$, defined in a neighborhood of zero, where

$$
I_{q}^{*}\left(\epsilon_{k}^{1}, \epsilon_{k}^{2}\left(\epsilon_{k}^{1}\right), \ldots, \epsilon_{k}^{m+1}\left(\epsilon_{k}^{1}\right)\right)=0 .
$$

By the Lagrange multiplier rule, there exist constants $\left(\omega_{q}\right)_{q=1,2, \ldots, m}$ such that

$$
\nabla\left(J(0, \ldots, 0)-\sum_{q=1}^{m} \omega_{q} I_{q}^{*}(0, \ldots, 0)\right)=(0, \ldots, 0) .
$$

For all $p=2, \ldots, n+1$, we have

$$
\begin{aligned}
\left(\frac{\partial J}{\partial \epsilon_{p-1}^{1}}\right)(0, \ldots, 0)= & \int_{x_{0}}^{x_{1}}\left(\partial_{p} L+\sum_{j=1}^{n}{ }_{x}^{R L} D_{x_{1}}^{\alpha_{p-1}^{j}} \partial_{n(p-1)+j+1} L\right. \\
& +\sum_{j=1}^{n}{ }_{x_{0}}^{R L} D_{x}^{\beta_{p-1}^{j}} \partial_{n(p-1)+n^{2}+j+1} L \\
& \left.+{ }_{x} I_{x_{1}}^{\gamma_{p-1}} \partial_{2 n^{2}+n+p} L\right) \sigma_{p-1}^{1}(x) d x
\end{aligned}
$$

and

$$
\begin{aligned}
\left(\frac{\partial I^{*}}{\partial \epsilon_{p-1}^{1}}\right)(0, \ldots, 0)= & \int_{x_{0}}^{x_{1}}\left(\partial_{p} F_{q}+\sum_{j=1}^{n}{ }_{x}^{R L} D_{x_{1}}^{\alpha_{p-1}^{j}} \partial_{n(p-1)+j+1} F_{q}\right. \\
& +\sum_{j=1}^{n}{ }_{x_{0}}^{R L} D_{x}^{\beta_{p-1}^{j}} \partial_{n(p-1)+n^{2}+j+1} F_{q} \\
& \left.+{ }_{x} I_{x_{1}}^{\gamma_{p-1}} \partial_{2 n^{2}+n+p} F_{q}\right) \sigma_{p-1}^{1}(x) d x
\end{aligned}
$$

Taking $E=L-\sum_{q=1}^{m} \omega_{q} F_{k}$, we obtain

$$
\begin{aligned}
& \int_{x_{0}}^{x_{1}}\left(\partial_{p} E+\sum_{j=1}^{n}{ }_{x}^{R L} D_{x_{1}}^{\alpha_{p-1}^{j}} \partial_{n(p-1)+j+1} E+\sum_{j=1}^{n}{ }_{x_{0}}^{R L} D_{x}^{\beta_{p-1}^{j}} \partial_{n(p-1)+n^{2}+j+1} E\right. \\
& \text { (40) } \left.\quad+{ }_{x} I_{x_{1}}^{\gamma_{p-1}} \partial_{2 n^{2}+n+p} E\right) \sigma_{p-1}^{1}(x) d x \\
& =0, \quad p=2, \ldots, n+1 \text {. }
\end{aligned}
$$


Using Lemma 5 of the calculus of variations, we can obtain:

$$
\begin{aligned}
\partial_{p} E & +\sum_{j=1}^{n}{ }_{x}^{R L} D_{x_{1}}^{\alpha_{p-1}^{j}} \partial_{n(p-1)+j+1} E \\
& +\sum_{j=1}^{n}{ }_{x_{0}}^{R L} D_{x}^{\beta_{p-1}^{j}} \partial_{n(p-1)+n^{2}+j+1} E+{ }_{x} I_{x}^{\gamma_{p-1}} \partial_{2 n^{2}+n+p} E \\
= & 0, \quad p=2, \ldots, n+1 .
\end{aligned}
$$

This ends the proof.

EXAMPLE 12. Now, we present an example of a fractional isoperimetric problem to illustrate the above theorem.

$$
\begin{aligned}
J\left(y_{1}, y_{2}, y_{3}\right)= & \int_{-1}^{1}\left(L \circ{ }_{\alpha_{k}^{j}}^{\gamma_{k}}\left[y_{1}, \ldots, y_{n}\right]_{\beta_{k}^{j}}\right)(x) d x \\
= & \int_{-1}^{1}\left(2 x^{2}+y_{1}^{2}+y_{2}^{2}+y_{3}^{2}\right. \\
& +2\left({ }_{-1}^{C} D_{x}^{\frac{1}{2}} y_{1}\right)^{2}-\left({ }_{-1}^{C} D_{x}^{\frac{3}{2}} y_{1}\right)^{2}+\frac{1}{2}\left({ }_{-1}^{C} D_{x}^{\frac{7}{3}} y_{1}\right)^{2} \\
& +3\left({ }_{-1}^{C} D_{x}^{\frac{1}{3}} y_{2}\right)^{2}+\frac{1}{2}\left({ }_{-1}^{C} D_{x}^{\frac{5}{3}} y_{2}\right)^{2}+\left({ }_{-1}^{C} D_{x}^{\frac{9}{4}} y_{2}\right)^{2} \\
& -\left({ }_{-1}^{C} D_{x}^{\frac{3}{4}} y_{3}\right)^{2}+3\left({ }_{-1}^{C} D_{x}^{\frac{5}{4}} y_{3}\right)^{2}+\frac{1}{4}\left({ }_{-1}^{C} D_{x}^{\frac{11}{5}} y_{3}\right)^{2} \\
& -{ }_{x}^{C} D_{1}^{\frac{6}{7}} y_{1}-{ }_{x} D_{1}^{\frac{9}{7}} y_{1}-{ }_{x} D_{1}^{\frac{15}{7}} y_{1} \\
& -2{ }_{x}^{C} D_{1}^{\frac{2}{5}} y_{2}+3{ }_{x}^{C} D_{1}^{\frac{7}{5}} y_{2}+{ }_{x}^{C} D_{1}^{\frac{14}{5}} y_{2} \\
& -{ }_{x}^{C} D_{1}^{\frac{3}{8}} y_{3}-\frac{1}{2}{ }_{x}^{C} D_{1}^{\frac{11}{8}} y_{3}-\frac{1}{2}{ }_{x}^{C} D_{1}^{\frac{17}{8}} y_{3} \\
& \left.+\frac{1}{3}-1 I_{x}^{\frac{5}{2}} y_{1}+2-{ }_{-1}^{\frac{7}{2}} I_{2}+{ }_{-1} I_{x}^{\frac{9}{2}} y_{3}\right) d x,
\end{aligned}
$$

subject to the boundary conditions:

$$
\begin{aligned}
& y_{1}(-1)=0 \text {, } \\
& y_{1}^{(1)}(-1)=-1, \\
& y_{1}^{(2)}(-1)=1 \text {, } \\
& y_{1}(1)=\sqrt{2} \text {, } \\
& y_{1}^{(1)}(1)=2 \text {, } \\
& y_{2}(-1)=-2 \text {, } \\
& y_{2}^{(1)}(-1)=0 \text {, } \\
& y_{1}^{(2)}(1)=\frac{3}{7} \text {, } \\
& y_{2}(1)=1 \text {, } \\
& y_{2}^{(1)}(1)=\frac{1}{2} \text {, } \\
& y_{3}(-1)=2 \sqrt{5} \text {, } \\
& y_{3}^{(1)}(-1)=-2 \text {, } \\
& y_{3}(1)=\frac{3}{4} \text {, } \\
& y_{3}^{(1)}(1)=0 \text {, } \\
& y_{2}^{(2)}(-1)=2 \sqrt{3} \text {, } \\
& y_{2}^{(2)}(1)=-1 \text {, } \\
& y_{3}^{(2)}(-1)=2 \text {, } \\
& y_{3}^{(2)}(1)=2 \text {, }
\end{aligned}
$$


and the fractional isoperimetric constraints:

$$
\begin{aligned}
I_{1}\left(y_{1}, y_{2}, y_{3}\right)= & \int_{-1}^{1}\left(F_{1} \circ{ }_{\alpha_{k}^{j}}^{\gamma_{k}}\left[y_{1}, \ldots, y_{n}\right]_{\beta_{k}^{j}}\right)(x) d x \\
= & \int_{-1}^{1}\left(x-2 y_{1}^{2}-y_{2}^{2}+y_{3}^{2}\right. \\
& -2\left({ }_{-1}^{C} D_{x}^{\frac{1}{2}} y_{1}\right)+\left({ }_{-1}^{C} D_{x}^{\frac{3}{2}} y_{1}\right)+4\left({ }_{-1}^{C} D_{x}^{\frac{7}{3}} y_{1}\right) \\
& -\left({ }_{-1}^{C} D_{x}^{\frac{1}{3}} y_{2}\right)^{2}+\frac{3}{2}\left({ }_{-1}^{C} D_{x}^{\frac{5}{3}} y_{2}\right)-\left({ }_{-1}^{C} D_{x}^{\frac{9}{4}} y_{2}\right) \\
& +2\left({ }_{-1}^{C} D_{x}^{\frac{3}{4}} y_{3}\right)+\left({ }_{-1}^{C} D_{x}^{\frac{5}{4}} y_{3}\right)^{2}-\left({ }_{-1}^{C} D_{x}^{\frac{11}{5}} y_{3}\right)^{2} \\
& +2{ }_{x}^{C} D_{1}^{\frac{6}{7}} y_{1}-2{ }_{x}^{C} D_{1}^{\frac{9}{7}} y_{1}+{ }_{x}^{C} D_{1}^{\frac{15}{7}} y_{1} \\
& -{ }_{x}^{C} D_{1}^{\frac{2}{5}} y_{2}-{ }_{x}^{C} D_{1}^{\frac{7}{5}} y_{2}+{ }_{x}^{C} D_{1}^{\frac{14}{5}} y_{2} \\
& +{ }_{x}^{C} D_{1}^{\frac{3}{8}} y_{3}-3{ }_{x}^{C} D_{1}^{\frac{11}{8}} y_{3}-4{ }_{x}^{C} D_{1}^{\frac{17}{8}} y_{3} \\
& \left.+\frac{1}{4}{ }_{-1} I_{x}^{\frac{5}{2}} y_{1}-22{ }_{-1} I_{x}^{\frac{7}{2}} y_{2}+3{ }_{-1}^{\frac{9}{2}} I_{3}\right) d x \\
= & 2,
\end{aligned}
$$

$$
\begin{aligned}
I_{2}\left(y_{1}, y_{2}, y_{3}\right)= & \int_{-1}^{1}\left(F_{2} \circ{ }_{\alpha_{k}^{j}}^{\gamma_{k}}\left[y_{1}, \ldots, y_{n}\right]_{\beta_{k}^{j}}\right)(x) d x \\
= & \int_{-1}^{1}\left(x^{2}-y_{1}^{2}+y_{2}^{2}+2 y_{3}^{2}\right. \\
& +\left({ }_{-1}^{C} D_{x}^{\frac{1}{2}} y_{1}\right)+2\left({ }_{-1}^{C} D_{x}^{\frac{3}{2}} y_{1}\right)-\left({ }_{-1}^{C} D_{x}^{\frac{7}{3}} y_{1}\right) \\
& +\left({ }_{-1}^{C} D_{x}^{\frac{1}{3}} y_{2}\right)^{2}+\left({ }_{-1}^{C} D_{x}^{\frac{5}{3}} y_{2}\right)+2\left({ }_{-1}^{C} D_{x}^{\frac{9}{4}} y_{2}\right) \\
& -\left({ }_{-1}^{C} D_{x}^{\frac{3}{4}} y_{3}\right)-\frac{1}{2}\left({ }_{-1}^{C} D_{x}^{\frac{5}{4}} y_{3}\right)^{2}+\left({ }_{-1}^{C} D_{x}^{\frac{11}{5}} y_{3}\right)^{2} \\
& +3{ }_{x}^{C} D_{1}^{\frac{6}{7}} y_{1}-{ }_{x}^{C} D_{1}^{\frac{9}{7}} y_{1}+\frac{1}{3}{ }_{x}^{C} D_{1}^{\frac{15}{7}} y_{1} \\
& -2{ }_{x}^{C} D_{1}^{\frac{2}{5}} y_{2}-{ }_{x}^{C} D_{1}^{\frac{7}{5}} y_{2}+{ }_{x}^{C} D_{1}^{\frac{14}{5}} y_{2} \\
& -{ }_{x}^{C} D_{1}^{\frac{3}{8}} y_{3}+{ }_{x}^{C} D_{1}^{\frac{11}{8}} y_{3}-{ }_{x}^{C} D_{1}^{\frac{17}{8}} y_{3} \\
& \left.+2{ }_{-1} I_{x}^{\frac{5}{2}} y_{1}-{ }_{-1} I_{x}^{\frac{7}{2}} y_{2}-{ }_{-1}^{\frac{9}{2}} I_{x}\right) d x \\
= & 3 .
\end{aligned}
$$


Here, $n=3, m=2, x_{0}=-1, x_{1}=1, \alpha_{1}^{1}=\frac{1}{2}, \alpha_{1}^{2}=\frac{3}{2}, \alpha_{1}^{3}=\frac{7}{3}, \alpha_{2}^{1}=\frac{1}{3}$, $\alpha_{2}^{2}=\frac{5}{3}, \alpha_{2}^{3}=\frac{9}{4}, \alpha_{3}^{1}=\frac{3}{4}, \alpha_{3}^{2}=\frac{5}{4}, \alpha_{3}^{3}=\frac{11}{5}, \beta_{1}^{1}=\frac{6}{7}, \beta_{1}^{2}=\frac{9}{7}, \beta_{1}^{3}=\frac{15}{7}, \beta_{2}^{1}=\frac{2}{5}$, $\beta_{2}^{2}=\frac{7}{5}, \beta_{2}^{3}=\frac{14}{5}, \beta_{3}^{1}=\frac{3}{8}, \beta_{3}^{2}=\frac{11}{8}, \beta_{3}^{3}=\frac{17}{8}, \gamma_{1}=\frac{5}{2}, \gamma_{2}=\frac{7}{2}, \gamma_{2}=\frac{9}{2}$, and

$$
E=L-\omega_{1} F_{1}-\omega_{2} F_{2} \text {. }
$$

By applying Eq. (34), we get the following fractional Euler-Lagrange equations:

$$
\begin{aligned}
2 y_{1} & +4 \omega_{1} y_{1}+2 \omega_{2} y_{1}+{ }_{x}^{R L} D_{1}^{\frac{1}{2}}\left(4{ }_{-1}^{C} D_{x}^{\frac{1}{2}} y_{1}+2 \omega_{1}-\omega_{2}\right) \\
& -{ }_{x}^{R L} D_{1}^{\frac{3}{2}}\left(2{ }_{-1}^{C} D_{x}^{\frac{3}{2}} y_{1}+\omega_{1}+2 \omega_{2}\right)+{ }_{x}^{R L} D_{1}^{\frac{7}{3}}\left({ }_{-1}^{C} D_{x}^{\frac{7}{3}} y_{1}-4 \omega_{1}+\omega_{2}\right) \\
& -{ }_{-1}^{R L} D_{x}^{\frac{6}{7}}\left(1+2 \omega_{1}+3 \omega_{2}\right)+{ }_{-1}^{R L} D_{x}^{\frac{9}{7}}\left(-1+2 \omega_{1}+\omega_{2}\right) \\
& -{ }_{-1}^{R L} D_{x}^{\frac{15}{7}}\left(1+\omega_{1}+\frac{1}{3} \omega_{2}\right)+{ }_{x} I_{1}^{\frac{5}{2}}\left(\frac{1}{3}-\frac{1}{4} \omega_{1}-2 \omega_{2}\right)=0,
\end{aligned}
$$

$$
\begin{aligned}
2 y_{2} & +2 \omega_{1} y_{2}-2 \omega_{2} y_{2}+{ }_{x}^{R L} D_{1}^{\frac{1}{3}}\left(6{ }_{-1}^{C} D_{x}^{\frac{1}{3}} y_{2}+2 \omega_{1}{ }_{-1}^{C} D_{x}^{\frac{1}{3}} y_{2}-2 \omega_{2}{ }_{-1}^{C} D_{x}^{\frac{1}{3}} y_{2}\right) \\
& +{ }_{x}^{R L} D_{1}^{\frac{5}{3}}\left({ }_{-1}^{C} D_{x}^{\frac{5}{3}} y_{2}-\frac{3}{2} \omega_{1}-\omega_{2}\right)+{ }_{x}^{R L} D_{1}^{\frac{9}{4}}\left(2{ }_{-1}^{C} D_{x}^{\frac{9}{4}} y_{2}+\omega_{1}-2 \omega_{2}\right) \\
(47) \quad+ & { }_{-1}^{R L} D_{x}^{\frac{2}{5}}\left(-2+\omega_{1}+2 \omega_{2}\right)+{ }_{-1}^{R L} D_{x}^{\frac{7}{5}}\left(3+\omega_{1}+\omega_{2}\right) \\
& +{ }_{-1}^{R L} D_{x}^{\frac{14}{5}}\left(1-\omega_{1}-\omega_{2}\right)+{ }_{x} I_{1}^{\frac{7}{2}}\left(2+2 \omega_{1}+\omega_{2}\right)=0,
\end{aligned}
$$

and

$$
\begin{aligned}
2 y_{3} & -2 \omega_{1} y_{3}-4 \omega_{2} y_{3}+{ }_{x}^{R L} D_{1}^{\frac{3}{4}}\left(-2{ }_{-1}^{C} D_{x}^{\frac{3}{4}} y_{3}-2 \omega_{1}+\omega_{2}\right) \\
& +{ }_{x}^{R L} D_{1}^{\frac{5}{4}}\left(6{ }_{-1}^{C} D_{x}^{\frac{5}{4}} y_{3}-2 \omega_{1}{ }_{-1}^{C} D_{x}^{\frac{5}{4}} y_{3}+\omega_{2}{ }_{-1}^{C} D_{x}^{\frac{5}{4}} y_{3}\right) \\
& +{ }_{x}^{R L} D_{1}^{\frac{11}{5}}\left(\frac{1}{2}{ }_{-1}^{C} D_{x}^{\frac{11}{5}} y_{3}+2 \omega_{1}{ }_{-1}^{C} D_{x}^{\frac{11}{5}} y_{3}-2 \omega_{2}{ }_{-1}^{C} D_{x}^{\frac{11}{5}} y_{3}\right) \\
& +{ }_{-1}^{R L} D_{x}^{\frac{3}{8}}\left(-1-\omega_{1}+\omega_{2}\right)+{ }_{-1}^{R L} D_{x}^{\frac{11}{8}}\left(-\frac{1}{2}+3 \omega_{1}-\omega_{2}\right) \\
& +{ }_{-1}^{R L} D_{x}^{\frac{17}{8}}\left(-\frac{1}{2}+4 \omega_{1}+\omega_{2}\right)+{ }_{x} I_{1}^{\frac{9}{2}}\left(1-3 \omega_{1}+\omega_{2}\right)=0 .
\end{aligned}
$$

4. Optimal Time Problem. Our aim here is to find the optimal time $T$ as well as the functions $\left(y_{k}\right)_{k=1,2, \ldots, n}$ that maximize or minimize the functional 
of the form:

$$
\begin{aligned}
J^{*}\left(y_{1}, \ldots, y_{n}, T\right)= & \int_{x_{0}}^{T} L^{*}\left(x, y_{1}(x), \ldots, y_{n}(x),\right. \\
& { }_{x_{0}} D_{x}^{\alpha_{1}^{1}} y_{1}(x), \ldots,{ }_{x_{0}} D_{x}^{\alpha_{1}^{n}} y_{1}(x), \ldots, \\
& \left.{ }_{x_{0}} D_{x}^{\alpha_{n}^{1}} y_{n}(x), \ldots,{ }_{x_{0}}^{C} D_{x}^{\alpha_{n}^{n}} y_{n}(x)\right) d x,
\end{aligned}
$$

where

$$
\begin{gathered}
\left(\left(y_{k}\right)_{k=1, \ldots, n}, T\right) \in B:=\left\{\left(C^{n}\left(\left[x_{0}, x_{1}\right]\right),\left[x_{0}, x_{1}\right]\right): y_{k}^{(i)}\left(x_{0}\right)=a_{k}^{i},\right. \\
\left.i=0,1, \ldots, n-1, n \in \mathbb{N}^{*}\right\} .
\end{gathered}
$$

We consider the both free and fixed end-point problems.

ThEOREM 13. Assume that $\left(\left(y_{k}\right)_{k=1, \ldots, n}, T\right) \in B$ is an extremizer of $J^{*}$ defined by Eq. (49). Then, $\left(\left(y_{k}\right)_{k=1, \ldots, n}, T\right)$ is a solution of the Euler-Lagrange equations:

$$
\partial_{p} L^{*}+\sum_{j=1}^{n}{ }_{x}^{R L} D_{T}^{\alpha_{p-1}^{j}} \partial_{n(p-1)+j+1} L^{*}=0, p=2, \ldots, n+1,
$$

for all $x \in\left[x_{0}, T\right]$, and satisfies the following transversality conditions:

1.

$$
\begin{gathered}
L^{*}\left(T, y_{1}(T), \ldots, y_{n}(T),{ }_{x}^{C} D_{T}^{\alpha_{1}^{1}} y_{1}(T), \ldots,{ }_{x}^{C} D_{T}^{\alpha_{1}^{n}} y_{1}(T),\right. \\
\left.\ldots,{ }_{x}^{C} D_{T}^{\alpha_{n}^{1}} y_{n}(T), \ldots,{ }_{x}^{C} D_{T}^{\alpha_{n}^{n}} y_{n}(T)\right)=0,
\end{gathered}
$$

2.

$$
\sum_{i=0}^{n-1} \sum_{j=1}^{n}{ }_{x}^{R L} D_{T}^{\alpha_{p-1}^{j}+i-n} \partial_{n(p-1)+j+1} L^{*}(x)=0, \text { at } x=T .
$$

Proof. Define

$$
\begin{aligned}
B^{*} & :=\left\{\sigma_{k} \in C^{n}\left(\left[x_{0}, x_{1}\right]\right): \sigma_{k}^{(i)}\left(x_{0}\right)=0, k=1, \ldots, n,\right. \\
i & =0,1, \ldots, n-1\} .
\end{aligned}
$$

Let $\sigma_{k} \in B^{*}$ and $y_{k}+\epsilon \sigma_{k}$ be variation of $y_{k}$, for all $k=1,2, \ldots, n$, and let $T+\epsilon \Delta T$ be variation of $T$, such that $\epsilon>0, \Delta T \in \mathbb{R}$.

Consider

$$
J^{*}(\epsilon)=J^{*}\left(y_{1}+\epsilon \sigma_{1}, \ldots, y_{n}+\epsilon \sigma_{n}, T+\epsilon \Delta T\right) .
$$


Assume that $\left(\left(y_{k}\right)_{k=1, \ldots, n}, T\right) \in B$ is an extremizer of $J^{*}$. Then, we get:

$$
\left(\frac{\partial J^{*}}{\partial \epsilon}\right)(\epsilon=0)=0 .
$$

Applying Leibniz integral rule, Eq. (56) implies that

$$
\begin{aligned}
& \int_{x_{0}}^{T}\left(\partial_{p} L^{*} \sigma_{p-1}(x)+\sum_{j=1}^{n} \partial_{n(p-1)+j+1} L^{*}{ }_{x_{0}}^{C} D_{x}^{\alpha_{p-1}^{j}} \sigma_{p-1}(x)\right) d x \\
& \quad+\Delta T L^{*}\left(T, y_{1}(T), \ldots, y_{n}(T),{ }_{x}^{C} D_{T}^{\alpha_{1}^{1}} y_{1}(T), \ldots,{ }_{x}^{C} D_{T}^{\alpha_{1}^{n}} y_{1}(T),\right. \\
& \left.\quad \ldots,{ }_{x}^{C} D_{T}^{\alpha_{n}^{1}} y_{n}(T), \ldots,{ }_{x}^{C} D_{T}^{\alpha_{n}^{n}} y_{n}(T)\right) \\
& =0, \quad p=2, \ldots, n+1 .
\end{aligned}
$$

Integrating the second term by parts as in Eq. (9) given in Lemma 6, we get:

$$
\begin{aligned}
\int_{x_{0}}^{T} & {\left[\partial_{p} L^{*}+\sum_{j=1}^{n}{ }_{x}^{R L} D_{T}^{\alpha_{p-1}^{j}} \partial_{n(p-1)+j+1} L^{*}\right] \sigma_{p-1}(x) d x } \\
& +\sum_{i=0}^{n-1} \sum_{j=1}^{n}\left[{ }_{x}^{R L} D_{T}^{\alpha_{p-1}^{j}+i-n}\left(\partial_{n(p-1)+j+1} L^{*}\right)(x)(-1)^{(n-i-1)} \sigma_{p-1}^{(n-i-1)}(x)\right]_{x=T} \\
& +\Delta T L^{*}\left(T, y_{1}(T), \ldots, y_{n}(T),{ }_{x}^{C} D_{T}^{\alpha_{1}^{1}} y_{1}(T), \ldots,{ }_{x}^{C} D_{T}^{\alpha_{1}^{n}} y_{1}(T),\right. \\
& \left.\ldots,{ }_{x}^{C} D_{T}^{\alpha_{n}^{1}} y_{n}(T), \ldots,{ }_{x}^{C} D_{T}^{\alpha_{n}^{n}} y_{n}(T)\right) \\
= & 0, \quad p=2, \ldots, n+1 .
\end{aligned}
$$

By fixing $\sigma_{p-1} \equiv 0, p=2, \ldots, n+1$, and by the arbitrariness of $\Delta T$, we obtain the first transversality condition. Then, the second transversality condition is proved by choosing $\sigma_{p-1}$ to be equal to zero on $\left[x_{0}, T\right)$ and $\sigma_{p-1}^{(n-i-1)}(T) \neq 0$, $p=2, \ldots, n+1, i=0,1, \ldots, n-1$. If, for all $p=2, \ldots, n+1, i=0,1, \ldots, n-1$, $\sigma_{p-1}^{(n-i-1)}$ is free on $[a, T)$ and $\sigma_{p-1}^{(n-i-1)}(T)=0$, we obtain Euler-Lagrange equation (51). This ends the proof.

EXAMPle 14. Consider the following optimal time problem:

$$
\begin{aligned}
J^{*}\left(y_{1}, y_{2}\right)= & \int_{-1}^{T}\left(2 x^{2}+\frac{1}{2} y_{1}^{2}+\frac{1}{2} y_{2}^{2}+2\left({ }_{-1}^{C} D_{x}^{\frac{2}{3}} y_{1}\right)\right. \\
& \left.+3\left({ }_{-1}^{C} D_{x}^{\frac{5}{4}} y_{1}\right)-\left({ }_{-1}^{C} D_{x}^{\frac{1}{2}} y_{2}\right)+2\left({ }_{-1}^{C} D_{x}^{\frac{7}{5}} y_{2}\right)^{2}\right) d x,
\end{aligned}
$$


with the boundary conditions:

$$
y_{1}(-1)=1, \quad y_{1}^{(1)}(-1)=\sqrt{2}, \quad y_{2}(-1)=-2, \quad y_{2}^{(1)}(-1)=-1 .
$$

We have: $n=2, x_{0}=-1, x_{1}=1, \alpha_{1}^{1}=\frac{2}{3}, \alpha_{1}^{2}=\frac{5}{4}, \alpha_{2}^{1}=\frac{1}{2}, \alpha_{2}^{2}=\frac{7}{5}$, and

$$
\begin{aligned}
L^{*}= & \left(2 x^{2}+\frac{1}{2} y_{1}^{2}+\frac{1}{2} y_{2}^{2}+2\left({ }_{-1}^{C} D_{x}^{\frac{2}{3}} y_{1}\right)\right. \\
& \left.-\left({ }_{-1}^{C} D_{x}^{\frac{5}{4}} y_{1}\right)^{2}-{ }_{-1}^{C} D_{x}^{\frac{1}{2}} y_{2}+2\left({ }_{-1}^{C} D_{x}^{\frac{7}{5}} y_{2}\right)^{2}\right) d x .
\end{aligned}
$$

By applying Eqs. (51), (52) and (53), we obtain the following fractional EulerLagrange equations:

$$
\left\{\begin{array}{c}
y_{1}+{ }_{x}^{R L} D_{T}^{\frac{2}{3}}(2)-2{ }_{x}^{R L} D_{T}^{\frac{5}{4}}\left({ }_{-1}^{C} D_{x}^{\frac{5}{4}} y_{1}\right)=0, \\
y_{2}-{ }_{x}^{R L} D_{T}^{\frac{1}{2}}(1)+4{ }_{x}^{R L} D_{T}^{\frac{7}{5}}\left({ }_{-1}^{C} D_{x}^{\frac{7}{5}} y_{2}\right)=0,
\end{array}\right.
$$

and for the transversality conditions, we get:

$$
\begin{gathered}
2 T^{2}+\frac{1}{2} y_{1}^{2}(T)+\frac{1}{2} y_{2}^{2}(T)+2\left({ }_{-1}^{C} D_{x}^{\frac{2}{3}} y_{1}\right)(T)-\left({ }_{-1}^{C} D_{x}^{\frac{5}{4}} y_{1}\right)^{2}(T) \\
-{ }_{-1}^{C} D_{x}^{\frac{1}{2}} y_{2}(T)+2\left({ }_{-1}^{C} D_{x}^{\frac{7}{5}} y_{2}\right)^{2}(T)=0 \\
{\left[{ }_{x} I_{T}^{\frac{4}{3}}(2)-2{ }_{x} I_{T}^{\frac{3}{4}}\left({ }_{-1}^{C} D_{x}^{\frac{5}{4}} y_{1}\right)+{ }_{x} I_{T}^{\frac{1}{3}}(2)-2{ }_{x}^{R L} D_{T}^{\frac{1}{4}}\left({ }_{-1}^{C} D_{x}^{\frac{5}{4}} y_{1}\right)\right]_{x=T}=0} \\
{\left[-{ }_{x} I_{T}^{\frac{3}{2}}(1)+4{ }_{x} I_{T}^{\frac{3}{5}}\left({ }_{-1}{ }^{C} D_{x}^{\frac{7}{5}} y_{2}\right)-{ }_{x} I_{T}^{\frac{1}{2}}(1)+4{ }_{x}^{R L} D_{T}^{\frac{2}{5}}\left({ }_{-1}^{C} D_{x}^{\frac{7}{5}} y_{2}\right)\right]_{x=T}=0,}
\end{gathered}
$$

where ${ }_{x}^{R L} D_{T}^{k}={ }_{x} I_{T}^{-k}$, for $k<0$.

\section{References}

1. Abdellaoui M.A., Dahmani Z., Solvability for a coupled system of nonlinear fractional integro-differential equations, Note Di Matematica, 35(1) (2015), 95-107.

2. Agrawal O.P., Generalized Euler-Lagrange equations and transversality conditions for FVPs in terms of the Caputo derivative, J. Vib. Control., 13(9-10) (2007), 1217-1237.

3. Agrawal O.P., Generalized variational problems and Euler-Lagrange equations, Comput. Math. Appl., 59(5) (2010), 1852-1864.

4. Almeida R., Fractional variational problems with the Riesz-Caputo derivative, Appl. Math. Lett., 25 (2012), 142-148.

5. Almeida R., Ferreira R. A. C., Torres D.F. M., Isoperimetric problems of the calculus of variations with fractional derivatives, Acta Mathematica Scientia, 32 (2012), 619-630. 
6. Almeida R., Malinowska A.B., Torres D.F.M., Fractional Euler-Lagrange differential equations via Caputo derivatives, in: Fractional dynamics and control, Springer, New York, 2012, 109-118.

7. Almeida R., Torres D.F.M., Calculus of variations with fractional derivatives and fractional integrals, Appl. Math. Lett., 22(12) (2009), 1816-1820.

8. Almeida R., Torres D. F. M., Necessary and sufficient conditions for the fractional calculus of variations with Caputo derivatives, Commun. Nonlinear Sci. Numer. Simul., 16 (2011), 1490-1500.

9. Almeida R., Tavares D., Torres D.F.M., The variable-order fractional calculus of variations, Springer, New York, 2019.

10. Anber A., Dahmani Z., The variational iteration method for solving the fractional coupled Lotka-Volterra equation, JIM., 14(4) (2011), 373-388.

11. Atanacković T. M., Konjik S., Pilipović S., Variational problems with fractional derivatives: Euler-Lagrange equations, J. Phys. A., 41(9) (2008), 1-12.

12. Bourdin L., Odzijewicz T., Torres D.F.M., Existence of minimizers for fractional variational problems containing Caputo derivatives, Adv. Dyn. Syst. Appl., 8 (2013), 3-12.

13. Brunt B. V., The calculus of variations, Universitext, Springer, New York, 2004.

14. Carathéodory C., Calculus of variations and partial differential equations of the first order, Chelsea, Providence, R.I., 1982.

15. Dahmani Z., Taïeb A., A coupled system of fractional differential equations involing two fractional orders, ROMAI Journal, 11(2) (2015), 141-177.

16. Dahmani Z., Taïeb A., New existence and uniqueness results for high dimensional fractional differential systems, Facta Nis Ser. Math. Inform., 30(3) (2015), 281-293.

17. Gelfand I. M., Fomin S. V., Calculus of variations, Moscow State University, Revised English Edition Translated and Edited by R. A. Silverman, Prentice-Hall, Inc., Englewood Cliffs, N.J., 1963.

18. Hilfer R., Applications of fractional calculus in physics, World Scientific, River Edge, New Jersey, 2000.

19. Kilbas A. A., Srivastava H. M., Trujillo J. J., Theory and applications of fractional differential equations, Elsevier B.V., Amsterdam, The Netherlands, 2006.

20. Klafter J., Lim S. C., Metzler R., Fractional dynamics, Recent Advances, World Scientific, Singapore, 2011.

21. Klimek M., On solutions of linear fractional differential equations of a variational type, The Publishing Office of the Czestochowa University of Technology, Częstochowa, 2009.

22. Malinowska A.B., Odzijewicz T., Torres D.F. M., Advanced methods in the fractional calculus of variations, Cham: Springer, 2015.

23. Malinowska A. B., Torres D. F. M., Generalized natural boundary conditions for fractional variational problems in terms of the Caputo derivative, Comput. Math. Appl., 59(9) (2010), 3110-3116.

24. Malinowska A.B., Torres D.F.M., Introduction to the fractional calculus of variations, Imperial College Press, London, 2012.

25. Miller K. S., Ross B., An introduction to the fractional calculus and fractional differential equations, Wiley, New York, 1993.

26. Oldham K. B., Spanier J., The fractional calculus, Academic Press, New York, 1974.

27. Riewe F., Nonconservative Lagrangian and Hamiltonian mechanics, Phys. Rev. E 3, 53(2) (1996), 1890-1899.

28. Samko S. G., Kilbas A. A., Marichev O. I., Fractional integrals and derivatives theory and applications, Gordon and Breach, Amsterdam, 1993. 
29. Taïeb A., Dahmani Z., A coupled system of nonlinear differential equations involving $m$ nonlinear terms, Georgian Math. Journal, 23(3) (2016), 447-458.

30. Taïeb A., Dahmani Z., The high order Lane-Emden fractional differential system: Existence, uniqueness and Ulam stabilities, Kragujevac Journal of Mathematics, 40(2) (2016), $238-259$.

31. Taïeb A., Dahmani Z., Some fractional variational problems involving Caputo derivatives, (Submitted).

32. Taïeb A., Existence of solutions and the Ulam stability for a class of singular nonlinear fractional integro-differential equations, Communications in Optimization Theory, 2019 (2019), Article ID 4, 1-22.

33. Taïeb A., Stability of singular fractional systems of nonlinear integro-differential equations, Lobachevskii Journal of Mathematics, 40(2) (2019), 219-229.

34. Weinstock R., Calculus of variations with applications to physics and engineering, Dover, New York, 1974.

35. Yourgrau W., Mandelstam S., Variational principles in dynamics and quantum theory, W. B. Saunders Company, Philadelphia, 1968.

36. Yousefi S. A., Dehghanb M., Lotfi A., Generalized Euler-Lagrange equations for fractional variational problems with free boundary conditions, Computers and Mathematics with Applications, 62 (2011), 987-995.

Received January 21, 2019

Revised June 19, 2019

Amele Taïeb

University of Mostaganem

LPAM, Faculty ST

UMAB

Algeria

e-mail: taieb5555@yahoo.com

Zoubir Dahmani

University of Mostaganem

LPAM, Faculty SEI

UMAB

Algeria

e-mail: zzdahmani@yahoo.fr 